\title{
Du folklore musical à l'ethnomusicologie. Entretien avec Diego Carpitella
}

Propos recueillis par Maurizio Agamennone

\section{Maurizio Agamennone et Diego Carpitella}

\section{(2) OpenEdition}

\section{Journals}

Édition électronique

URL : http://journals.openedition.org/ethnomusicologie/1595

ISSN : 2235-7688

\section{Éditeur}

ADEM - Ateliers d'ethnomusicologie

\section{Édition imprimée}

Date de publication : 1 octobre 1991

Pagination : 229-238

ISBN : $978-2-8257-0437-8$

ISSN : 1662-372X

\section{Référence électronique}

Maurizio Agamennone et Diego Carpitella, « Du folklore musical à l'ethnomusicologie. Entretien avec Diego Carpitella », Cahiers d'ethnomusicologie [En ligne], 4 | 1991, mis en ligne le 01 janvier 2012, consulté le 10 décembre 2020. URL : http://journals.openedition.org/ethnomusicologie/1595

Ce document a été généré automatiquement le 10 décembre 2020.

Tous droits réservés 


\section{Du folklore musical à l'ethnomusicologie. Entretien avec Diego Carpitella*}

Propos recueillis par Maurizio Agamennone

Maurizio Agamennone et Diego Carpitella

Le 7 août 1990, Diego Carpitella est décédé des suites d'un grave infarctus, laissant un grand vide dans la culture musicale italienne. Il naquit le 12 juin 1924, à Reggio Calabria, dans l'extrême sud de la péninsule italienne, une terre qui fut longtemps le lieu privilégié de nombre de ses recherches. Ses élèves, ses amis et ses collègues l'ont salué pour la dernière fois à l'occasion des funérailles qui ont eu lieu le 9 août 1990 près de la Faculté des lettres et de philosophie de l'Université La Sapienza de Rome, où il avait travaillé pendant quinze ans. Son œuvre de savant a été évoquée récemment en Italie à l'occasion de deux réunions internationales, à la conception et à la promotion desquelles il avait activement participé, toutes deux sur des thèmes qui avaient fortement caractérisé son activité (La musique et le cinéma, Sienne, août 1990 ; Tendances de la recherche musicologique, Latina, septembre 1990). En septembre 1991, à l'initiative de l'Université du Basilicate (région où Carpitella avait fait ses débuts comme chercheur) sont organisées à Potenza des journées d'étude placées sous le titre Sources écrites et orales de la musique du Mezzogiorno - Hommage à Diego Carpitella, avec la participation de nombreux chercheurs d'orientations diverses (ethnomusicologie, histoire de la musique, ethnologie), occupés à restituer les expressions musicales du sud italien.

Dans les premiers jours de juillet 1990, quelques semaines avant sa disparition, nous avons recueilli cet entretien pour les Cahiers de musiques traditionnelles, où Diego Carpitella décrivit ses propres expériences de recherche et exprimait quelques opinions sur les plus récentes questions de la recherche ethnomusicologique, ainsi que certaines idées sur les vicissitudes et les perspectives de l'ethnomusicologie italienne. Étant donné les circonstances dans lesquelles elles furent recueillies, ces idées représentent, probablement, la dernière manifestation de la pensée de Carpitella. C'est pourquoi elles constituent aujourd'hui un témoignage particulièrement précieux et émouvant. 
3 Après avoir recueilli de nombreux documents sonores pour le Centre national d'études de musique populaire (CNSMP) de l'Académie nationale de Sainte Cécile à Rome au début des années cinquante, Diego Carpitella participa comme ethnomusicologue aux recherches ethnographiques d'Ernesto De Martino sur la lamentation funèbre et sur la thérapie du tarentisme en Italie méridionale dans les années cinquante et soixante. Pendant la même période, il réalisa avec Alan Lomax le premier recueil systématique d'enregistrements qui constitue le corpus de documents sonores le plus remarquable sur la musique traditionnelle italienne. Par la suite, il entreprit une intense activité d'enseignement qui devait culminer, en 1976, par l'institution, à l'Université La Sapienza de Rome, de la première chaire d'ethnomusicologie dans les universités italiennes, dont Carpitella est resté titulaire jusqu'à ses derniers jours. En même temps, il a développé un très vif intérêt pour l'anthropologie et l'ethnomusicologie visuelles: il fut président de l'Association italienne de cinématographie scientifique (AICS). De 1970 à 1983, il dirigea l'Institut d'histoire des traditions populaires de l'Université La Sapienza. De 1977 à 1989, il dirigea les Séminaires internationaux d'ethnomusicologie de l'Accademia Musicale Chigiana à Sienne, auxquels ont participé, depuis plus de dix ans, les plus grands savants européens, américains et asiatiques: sur la base de cette expérience, l'Accademia Musicale Chigiana a organisé et accueilli à Sienne la recontre du Séminaire européen d'ethnomusicologie en août 1989. Diego Carpitella a en outre encouragé la constitution de la Société italienne d'ethnomusicologie (SIE) qu'il présida jusqu'en 1987 ; il a également fondé et dirigé la revue Culture musicali - Quaderni di etnomusicologia. Enfin, quelques mois avant sa disparition, il fut nommé Conservateur des Archives d'ethnomusicologie de L'Académie nationale de Sainte Cécile à Rome.

M.A.

Diego Carpitella (1924-1990).

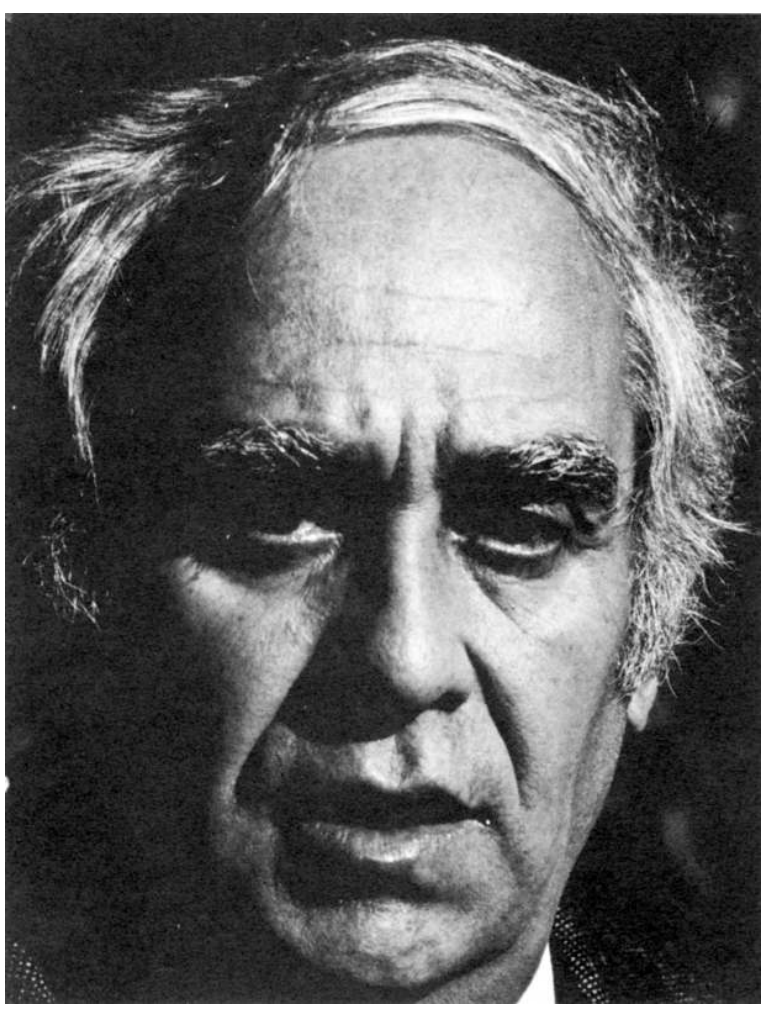

Cahiers d'ethnomusicologie, 4 | 1991 
Maurizio Agamennone: Vous êtes considéré comme un protagoniste de l'ethnomusicologie italienne. Comment a commencé votre activité de savant et de chercheur?

Diego Carpitella : J'ai commencé à travailler en 1950-52 au Centre national d'études de musique populaire de l'Académie nationale de Sainte Cécile, en enregistrant des milliers de documents sonores avec les moyens techniques de la Radio-télévision italienne (RAI). Par la suite, j'ai rencontré Ernesto De Martino et, m'occupant des expressions musicales, j'ai participé avec son équipe aux recherches sur la lamentation funèbre dans la culture traditionnelle de l'Italie méridionale, qui s'inscrivait dans le cadre d'une étude plus étendue sur la lamentation rituelle dans le monde antique ${ }^{1}$. Puis ce fut le tour d'Alan Lomax en 1954-55: nous avons réalisé la première exploration sonore systématique sur le territoire italien, en parcourant ensemble l'Italie en long et en large pendant près d'une année ; le résultat de ce voyage consista en deux disques microsillons qui furent publiés aux États-Unis en 1957 et ne parurent en édition italienne que seize ans plus tard. En 1959, toujours dans l'équipe interdisciplinaire d'Ernesto De Martino, j'ai participé aux recherches sur l'exorcisme choréo-musical du tarentisme des Pouilles, avec de nombreux enregistrements sonores : c'est aussi à cette occasion que j'ai réalisé mon premier document filmé en $16 \mathrm{~mm}$.

M.A. : Comment s'est poursuivie votre activité, surtout en ce qui concerne les recherches sur le terrain et la définition des études ethnomusicologiques italiennes ?

D.C. : Au cours des années soixante et soixante-dix, j'ai effectué des recherches auprès des communautés italiennes en Amérique latine (Brésil, Argentine, Uruguay), et je me suis occupé de la réalisation d'un ensemble de documents sonores sur la musique paysanne de l'Arentin en Toscane. En 1970-71, avec Tokhariev, j'ai participé à quelques recherches en URSS, comme conseiller ethnographique. En 1973-74, j'ai commencé à travailler de façon plus intense dans l'anthropologie et l'ethnomusicologie visuelles, en réalisant de nombreux films dans diverses régions d'Italie.

En ce qui concerne l'enseignement, j'ai donné des cours d'histoire de la musique et d'ethnomusicologie aux Universités de Chieti et de Trente; et pendant plusieurs années, un cours sur l'histoire et la technique du chant populaire au Conservatoire de musique Sainte Cécile à Rome, cela jusqu'en 1976, époque à laquelle j'ai pris la chaire d'ethnomusicologie à l'Université La Sapienza de Rome. Auparavant, en 1973, j'avais organisé la première réunion consacrée aux études ethnomusicologies en Italie, qui a abouti, en 1976, à la création de la Société italienne d'ethnomusicologie et, par là même, à la publication de la revue Culture musicali. J'ai aussi dirigé des collections de livres ${ }^{2}$, de disques $^{3}$ et de vidéo ${ }^{4}$.

Enfin, je voudrais rappeler les Séminaires internationaux d'ethnomusicologie de l' Accademia Musicale Chigiana de Sienne: chaque année, depuis 1977, de nombreux savants y ont participé, dont Simha Arom, John Blacking, Jean During, Anca Giurchescu, Mantle Hood, Janos Karpati, Roberto Leydi, Bernard Lortat-Jacob, Jean Jacques Nattiez, Gilbert Rouget, Ivan Vandor et Hugo Zemp.

M.A. : A propos de recherche sur le terrain et de documentation, dans quelle mesure ce que l'on appelle l'ethnologie d'urgence a intéressé les enquêtes réalisées en Italie?

D.C.: La question de l'ethnologie d'urgence concerne les rapports entre sociétés complexes et sociétés traditionnelles. Une partie de notre planète (peut-être même la plus grande) s'est transformée si rapidement qu'elle court le risque de perdre son identité, du moins en ce qui concerne le passé, la tradition. Sur le territoire italien, en 
une trentaine d'années, de nombreuses formes de l'expressivité de tradition orale se sont éclipsées ou ont disparu. Les enregistrements systématiques réalisés depuis 1948 ont tenté de recueillir les expressions du monde aux racines dites agro-pastorales. Avec le temps, ces documents deviennent toujours plus rares et impossibles à répéter. Parmi les musiques avec tambourin (tamburello) et cornemuse (zampogna) existant en Calabre et en Lucanie, certaines ont désormais "perdu » leurs caractéristiques particulières dans le domaine du rythme et de l'intonation, ainsi que dans la façon d'accompagner la voix, elle aussi toujours plus difficile à recueillir dans le « style ancien ».

M.A. : Un des centres d'intérêt les plus remarquables de vos recherches est l'étude des états de possession. Quelle a été votre expérience?

D.C. : Si je n'avais pas eu l'expérience du terrain dans l'étude du tarentisme des Pouilles, je n'aurais pas pu m'occuper ensuite du stambali tunisien ni du chamanisme en Amazonie ; c'est-à-dire que la méthodologie interdisciplinaire adoptée au cours de ces recherches de 1959 à 1960 dépassait l'aspect purement local de l'événement; surtout en ce qui concerne les liens entre induction musicale et ce qu'on appelle les états altérés de conscience: les connexions entre sons et cinétique rituelle qui sont au centre des états de possession, au-delà de l'idéologie du rite. Dans le stambali tunisien, d'origine soudanaise, l'état altéré de la conscience, tel que je l'ai vu, est moins dramatique, plus "quotidien", plus un divertissement, propre à susciter une inévitable comparaison entre «folie» et poésie. L'expérience du tarentise m'a beaucoup servi pour repérer avec une plus grande sûreté les états « canoniques » et les états « paroxystiques » des chamanes. Les cris, les sifflements, les cantilènes des Yanoama au cours des rites nocturnes m'ont souvent fait penser à la chapelle de San Paolo à Galatina où l'on résolvait la crise du tarentisme.

M.A. : Vous êtes président de l'Association italienne de cinématographie scientifique. Quelle signification attribuez-vous à l'anthropologie visuelle?

D.C. : La même importance que j'attribue à l'enregistrement sonore pour la musique. On sait bien que les sons, comme les images en mouvement, ne sont pas exprimables avec les mots. Si le dessin statique parvient à suggérer la figura fluens, il ne la rend pas pour autant dans sa substance documentaire. De même, si la représentation des sons sur la portée n'est pas entièrement exhaustive pour la musique savante européenne, imaginez ce qu'elle peut être pour les cultures non européennes! Au début de mes recherches, j'ai surtout été attiré par la "pure » cinétique gestuelle, mais par la suite, mon intérêt s'est porté avant tout sur la somatisation du son (ou, si vous voulez, aussi le contraire, la sonorisation du corps). Dans le contexte rituel, cette synesthésie est déterminante, comme l'a magistralement démontré Marcel Mauss et comme l'a confirmé Gilbert Rouget dans son livre La musique et la transe ${ }^{5}$.

M.A.: Quelles sont, plus particulièrement, vos expériences de terrain en matière d'anthropologie visuelle?

D.C. : Ma première expérience fut de filmer des «soins à domicile» pour une femme atteinte de tarentisme dans un petit hameau ensoleillé de la province de Lecce. C'était la première fois que j'utilisais une caméra $16 \mathrm{~mm}$, n'étant pas opérateur professionnel, ni même amateur. Mais j'avais néanmoins amené avec moi la caméra, parce qu'elle me semblait être le seul instrument valable pour enregistrer l'événement. Quant à la synchronisation entre images et son, je l'ai effectuée par la suite. Puis j'ai filmé la gestuelle de Naples, idée qui m'est venue à la lecture d'un texte publié à Naples en 1832 par le chanoine De Jorio ${ }^{6}$. Ensuite, pour comparer, je me suis occupé de la cinétique 
chez les bergers de Barbagia (région intérieure de la Sardaigne), que je rapprochai peut-être un peu arbitrairement - des statues nouragiques (VIII ${ }^{\mathrm{e}}-\mathrm{VII}{ }^{\mathrm{e}}$ siècle av. J.-C.) et du micro-système musical sarde que j'avais étudié auparavant. J'ai aussi réalisé des documents filmés - toujours en $16 \mathrm{~mm}$ - sur certains musiciens de Calabre, de Sardaigne et de la plaine du Pô. Plus tard, dans le cadre de recherches sur l'organologie en Calabre, promues par le Département d'études linguistico-anthropologiques de l'Université, nous avons réalisé un moyen métrage vidéo au sujet d'un court traité sur les instruments populaires, découvert près de Capo Vaticano, dans la province de Catanzaro. Sous le titre I quaderni di Reginaldo, cette bande vidéo présente une centaine de dessins d'instruments de musique de tradition populaire, aujourd'hui pratiquement disparus ; il s'agit d'un vrai traité d'ethno-organologie.

M.A. : Quels moyens technologiques préférez-vous employer aujourd'hui dans vos travaux d'anthropologie visuelle?

D.C. : Ce n'est pas seulement un problème d'ordre technologique. Il s'agit de définir comment et pourquoi utiliser l'objectif et le microphone. Jean Rouch affirme qu'on dispose aujourd'hui d'une telle quantité de moyens techniques sophistiqués que l'anthropologue risque tout simplement de ne pas savoir comment s'en servir - non pas parce qu'on serait incapable de les manier et de les faire fonctionner, mais faute de l'aplomb et de l'ouverture d'esprit nécessaires pour y parvenir. La haute définition est encore dans la pellicule, mais l'électronique entraîne tant d'avantages (notamment économiques) qu'on ne saurait la négliger. Quant à l'enregistrement digital du son, il est en train de s'imposer autant sur le terrain qu'en studio.

M.A.: Pour revenir sur un problème d'ordre théorique: d'après votre expérience, l'ethnomusicologie se fonde-t-elle sur un critère géographique ou fait-elle plutôt appel à une spécificité méthodologique?

D.C.: C'est là un thème central dans le dialogue entre musicologues et ethnomusicologues. Quant à moi, je préfère la définition historique de musicologie comparée, car au lieu d'entraîner un compartimentage, elle examine des formes culturelles susceptibles d'être comparées. Une musicologie qui ne tient pas compte du contexte dans lequel se déroule l'événement musical, de la «langue » qui pèse sur les épaules du musicien ou encore des niveaux sociaux et culturels auxquels se produit ou se consomme la musique, est une musicologie «boiteuse», D'autre part, une ethnomusicologie qui mène de façon insensée à une idéologie vague, sans se préoccuper de la «structure» des sons, me semble tout aussi défaillante. Quoiqu'il en soit, il est clair que si l'on examine des zones culturelles différentes, on doit sélectionner et affiner les critères et les paramètres d'analyse, tout en évitant que la spécialisation se transforme automatiquement en un ghetto.

M.A.: Une des motivations les plus fortes pour de nombreux spécialistes actuels est justement le repérage des homologies dans l'expérience musicale, au-delà des particularités que l'on peut déceler dans les différentes cultures...

D.C. : ...c'est une question qui intéresse toute l'anthropologie. On s'est détourné de la différence et de sa mise en valeur pour se concentrer, aujourd'hui, sur les analogies, les traits communs d'identité. Encore les universaux! A ce propos, c'est l'ethnoscience qui a réussi une des percées les plus significatives de ces dernières années: en prenant connaissance de la diversité des modes de pensée, on tient en échec l'ethnocentrisme, sans oublier que la diversité relève souvent du point de vue étique, ce qui la rend, en fait, idéologiquement discutable. Le repérage d'analogies universelles dérive souvent de 
la confrontation de connaissances obtenues actuellement à l'aide de technologies plus sophistiquées qui, lorsqu'elles sont maniées par « les autres », désamorcent l'altérité.

En ce sens, une musicologie différente, transculturelle et embrassant tous les domaines, pourrait abattre la cloison qui sépare encore musicologie et ethnomusicologie.

M.A.: Pour terminer, l'ethnomusicologie italienne se définit-elle comme une tradition d'expériences "démo-musicologiques», tournée en grande partie vers les musiques populaires de l'Italie, ou bien, selon votre expérience, I'horizon des études est-il plus vaste?

D.C.: C'est une insinuation qu'on entend parfois à propos de l'ethnomusicologie italienne, mais elle est infondée. L'histoire et la culture italiennes sont très particulières. L'Italie est un territoire historique qui présente de hauts sommets de culture savante mais aussi de profondes racines folkloriques qui ont eu une grande influence culturelle. Les cris et les chants des marchands ambulants, l'appel des animaux, la lamentation funèbre, les formes de polyphonie, les modalités d'échelles, les styles de chant, les musiques instrumentales et les techniques de construction de certains instruments de musique, les formes de danse, les modes de transmission du savoir musical: autant d'objets appartenant pleinement au domaine de l'ethnomusicologie. Si cela n'est pas très connu, c'est parce que l'édition ethnomusicologique italienne est encore maigre.

Si, au contraire, il faut comprendre par ethnomusicologie une discipline qui étudie " uniquement » les musiques extra-européennes de tradition orale... alors je ne suis pas d'accord avec cette façon restrictive de comprendre l'ethnomusicologie.

Toutefois, pour ne pas en rester là, je tiens à signaler un numéro double de Culture musicali ${ }^{7}$ entièrement consacré aux résultats de recherches récentes menées par des ethnomusicologues italiens au Cambodge, à Madagascar, au Népal, au Zaïre et au Maroc.

\section{Principales publications de Diego Carpitella}

\section{Bibliographie}

1952, « Gli studi sul folklore musicale in Italia ». Società VIII(3) : 1-11.

1956a, « Problemi attuali della musica popolare in Italia ». Ricordiana II(9) : 417-21.

1956b, Ritmi e melodie di danze popolari in Italia. Roma : Accademia Nazionale di Santa Cecilia.

1958, « Etnomusicologia e culture musicale ». La Rassegna musicale XXVIII(3) : 204-8.

1961a, « L'esorcismo coreutico-musicale del tarantismo ». In : E. De Martino. La terra del rimorso.

Milano : Il Saggiatore, pp. 335-71. 
1961b, « Folk music (Italian) ». In : Grove's Dictionary of Music and Musicians. Suppl. volume. London : McMillan, pp. 135-54.

1961c, « Il mito del primitivo nella musica contemporanea ». Terzo Programma : 217-56.

1966a, « Studio etnomusicologico della documentazione sonora Yanoama ». In : E. Biocca, Viaggi tra gli Indi, vol. III. Roma : Centro Nazionale delle Ricerche, pp. 267-91.

1966b, « America. Stati Uniti. Musica popolare ». In : La Musica. I vol. , II partie. Torino : Unione Tipografica Editrice Torinese, pp. 46-56.

1966c, « Europa (Etnomusicologia) ». In : La Musica. II vol. , I partie. Torino : Unione Tipografica Editrice Torinese, pp. 371-86.

1967, « La musica nei rituali dell'argia ». In : C. Gallini, I rituali dell'argia. Padova : Cedam, pp. 293-307.

1971, Motivi critici negli studi di folklore musicale in Italia dal 1945 ad oggi. Roma : Bulzoni.

1973, Musica e tradizione orale. Palermo : Flaccovio.

1975a, L'etnomusicologia in Italia (ed.). Palermo : Flaccovio.

1975b, « Der Diaulos des Celestino ». Die Musikforschung XXVIII (4) : 422-428

1976, Folklore e analisi differenziale di cultura. Roma : Bulzoni.

1977a, Musica contadina dell'Arentino. Roma : Bulzoni.

1977b, « Informazione e ricerca nel film etnografico italiano (1950-1976) ». Roma : Associazione Italiana di Cinematografia Scientifica, pp. 15-16.

1978, « Confronti binari e captatio demologica ». In : Letteratura popolare brasiliana e tradizione europea. Roma : Bulzoni, pp. 231-36.

1979, « Jazz ». In : Enciclopedia del Novecento, vol. III. Roma : Istituto dell’Enciclopedia Italiana, pp. 913-24.

1983, « Etnomusicologia ». In : Dizionario Enciclopedico Universale della Musica e dei Musicisti. Il lessico, vol. II. Torino : Unione Tipografica Editrice Torinese, pp. 184-88.

1985, « Dal mito del primitivo all'informazione interculturale nella musica moderna ». Studi musicali XIV(1) : 193-208.

1986, « Suono e immagine ». In : Intervista strumento di comunicazione. Roma : Discoteca di Stato, pp. 112-16.

1989a, « La cinesica di San Vivaldo ». In : Gerusalemme di San Vivaldo e i Sacri Monti in Europa. Montaione : Pacini, pp. 289-97.

1989b, « La trasmissionne del sapere : la respirazione circolare nelle "launeddas” sarde ». In : G.R. Cardona, La trasmissione del sapere : aspetti linguistici e antropologici. Roma : Bagatto-libri, pp. 99-110.

1989c, Ethnomusicologica. Seminari internazionali di Etnomusicologia, 1977-1989 (ed.). Siena : Accademia Musicale Chigiana. 


\section{Discographie}

Northern and Central Italy and the Albanians of Calabria, par Alan Lomax et Diego Carpitella. Disque $30 \mathrm{~cm} 33 \mathrm{t}$. The Columbia World Library of Folk and Primitive Music, vol. XV. Columbia KL 5173, 1957.

Southern Italy and the Islands, par Alan Lomax et Diego Carpitella. Disque $30 \mathrm{~cm} 33 \mathrm{t}$. The Columbia World Library of Folk and Primitive Music, vol. XVI. Columbia KL 5174, 1957.

Music and Song of Italy. Recorded in the field by Alan Lomax and Diego Carpitella, edited by Alan Lomax. Disque $30 \mathrm{~cm} 33$ t. Tradition Records TLP 1030, 1958.

Italian Folk Music, vol. 1 : Piedmont, Emilia, Lombardy. Recorded in the field by Alan Lomax with Diego Carpitella. Disque $30 \mathrm{~cm} 33$ t. Ethnic Folkways Records FE 4271, 1972.

Italian Folk Music, vol. 5 : Naples, Campania, par Alan Lomax, Diego Carpitella and Carla Bianco. Disque $30 \mathrm{~cm} 33$ t. Ethnic Folkways Records FE 4265, 1972.

Musica sarda, par Diego Carpitella, Pietro Sassu, Leonardo Sole. 3 disques $30 \mathrm{~cm} 33 \mathrm{t}$. Albatros VPA 8150-8152, 1973.

Folklore musicale italiano, par Alan Lomax et Diego Carpitella. 2 disques $30 \mathrm{~cm} 33$ t. Pull QLP 107-108, 1973.

Documenti dell'Archivio Etnico Linguistico Musicale della Discoteca di Stato, par Antonino Pagliaro e Diego Carpitella. 2 disques $30 \mathrm{~cm} 33$ t. Roma : Discoteca di Stato, DdS 2G3KP 1931-2, 2G3KY 1933-4, 1974.

Musica contadinadell'Aretino. 3 disques $30 \mathrm{~cm} 33$ t. Albatros VPA 8286-8288, 1976.

Yanoama : tecniche vocali dello sciamanismo, par Ettore Biocca et Diego Carpitella. Disque $30 \mathrm{~cm} 33 \mathrm{t}$. Cetra SU 5003, 1979.

\section{Filmographie}

Meloterapia del tarantismo (Les Pouilles). 14 minutes. $16 \mathrm{~mm}, \mathrm{~b} / \mathrm{n}$, son. Produit par Diego Carpitella, 1960.

Cinesica culturale 1. Napoli. 39 minutes. 16 mm, couleurs, son. Roma : Istituto Luce, 1973.

Cinesica culturale 2. Barbagia (Sardaigne). 41 minutes. $16 \mathrm{~mm}$, couleurs, son. Roma : Istituto Luce, 1974.

Il doppio flauto di Celestino (Montemarano. Campanie). 8 minutes. 3/4 U Matic, b/n, son. Documentazione Folklorica Audiovisiva, Gruppo di ricerca dell'Istituto di Storia delle tradizioni popolari. Roma : Università degli studi « La Sapienza », 1975.

Cinesica culturale 4. Materiali sul Palio (Sienne, Toscane), I et II partie. 25, 30 minutes. $16 \mathrm{~mm}$, couleurs, son. Produit par le Centro per le tradizioni popolari. « Fondazione Lavoratori Officine Galilei ». Firenze : Regione Toscana, 1979.

Sardegna : is launeddas. 34 minutes. $16 \mathrm{~mm}$, couleurs, son. Produit par RAI III ${ }^{a}$ Rete TV. Roma : RAI Radiotelevisione italiana, 1982.

Calabria : zampogna e chitarra battente. 31 minutes. $16 \mathrm{~mm}$, couleurs, son. Produit par RAI III ${ }^{\text {Rete }}$ TV. Roma : Radiotelevisione italiana, 1982. 
Emilia : Brass Band della Padana (Parme, Emilie). 31 minutes. $16 \mathrm{~mm}$, couleurs, son. Produit par RAI III Rete TV. Roma : Radiotelevisione italiana, 1982.

I Quaderni di Reginaldo (Spilinga, Calabre). 29.30 minutes, VHS 1/2, couleurs, son. Produit par le Dipartimento di studi glotto-antropologici/Etnomusicologia. Roma : Università degli studi « La Sapienza », 1988.

\section{NOTES}

*. Traduit de l'italien par Georges Goormaghtigh.

1. Ernesto De Martino, Morte e pianto rituale. Dal lamento funebre antico al pianto di Maria. Boringhieri, 1975 [ $1^{\text {re }}$ éd. en 1958]. Pour les publications de Carpitella, voir infra.

2. Dans la Collana di etnomusicologia (Rome, Bulzoni), dirigée par Carpitella, cinq volumes ont déjà été publiés.

3. Carpitella se réfère à la collection de disques microsillons $30 \mathrm{~cm}, 33 \mathrm{t}$. I Suoni - Musica di tradizione orale, publiée par Fonit-Cetra et consacrée en majeure partie à la musique traditionnelle italienne.

4. A partir de 1988, sur l'initiative de la chaire d'ethnomusicologie de l'Université La Sapienza de Rome, en collaboration avec la Discothèque de l'État et l'Association italienne de cinématographie scientifique, dans la série appelée MIV Musica Identità Video, furent produites huit monographies consacrées pour la plupart à des instruments de musique et à des rituels de l'Italie méridionale.

5. Gilbert Rouget, La musique et la transe. Esquisse d'une théorie générale des relations de la musique et de la possession. Paris : Gallimard, 1980 [rééd. en 1990].

6. Andrea De Jorio, La mimica degli antichi investigata nel gestire napoletano. Naples: Associazione napoletana per i monumenti e il paesaggio, 1964.

7. Culture musicali : «Etnomusicologia italiana fuori di casa », V/VI(10-11), 1986-87. Florence : La Casa Usher. 